\title{
Anomalous effective medium approximation breakdown in deeply subwavelength all- dielectric photonic multilayers
}

\author{
Andryieuski, Andrei; Lavrinenko, Andrei; Zhukovsky, Sergei
}

Published in:

Nanotechnology

Link to article, DOI:

$10.1088 / 0957-4484 / 26 / 18 / 184001$

Publication date:

2015

Document Version

Peer reviewed version

Link back to DTU Orbit

Citation (APA):

Andryieuski, A., Lavrinenko, A., \& Zhukovsky, S. (2015). Anomalous effective medium approximation breakdown in deeply subwavelength all-dielectric photonic multilayers. Nanotechnology, 26(18), [184001].

https://doi.org/10.1088/0957-4484/26/18/184001

\section{General rights}

Copyright and moral rights for the publications made accessible in the public portal are retained by the authors and/or other copyright owners and it is a condition of accessing publications that users recognise and abide by the legal requirements associated with these rights.

- Users may download and print one copy of any publication from the public portal for the purpose of private study or research.

- You may not further distribute the material or use it for any profit-making activity or commercial gain

- You may freely distribute the URL identifying the publication in the public portal

If you believe that this document breaches copyright please contact us providing details, and we will remove access to the work immediately and investigate your claim. 


\title{
Anomalous effective medium approximation breakdown in deeply subwavelength all-dielectric photonic multilayers
}

\author{
Andrei Andryieuski ${ }^{1}$, Andrei V Lavrinenko ${ }^{1}$, and Sergei V Zhukovsky ${ }^{1,2}$ \\ ${ }^{1}$ DTU Fotonik, Technical University of Denmark, Ørsteds pl. 343, Kongens Lyngby, 2800 Denmark \\ ${ }^{2}$ ITMO University, Kronverkskiy pr. 49, St. Petersburg, 197101 Russia \\ E-mail: $\underline{\text { sezh@fotonik.dtu.dk }}$
}

\begin{abstract}
We present a comprehensive analysis of the applicability of the effective medium approximation to deeply subwavelength (period $\leq \lambda / 50$ ) all-dielectric multilayer structures. We demonstrate that even though the dispersion relations for such multilayers differ from the effective medium prediction only slightly, there can be regimes when an actual multilayer stack exhibits significantly different properties compared to its homogenized model. In particular, reflection near the critical angle is shown to strongly depend on even very small period variations, as well as on the choice of the multilayer termination. We identify the geometries for which the influence of the subwavelength features is maximized and demonstrate that the difference between the reflectance from the actual multilayer and the effective medium prediction can be as great as 0.98 . The results of this analysis can be useful for high-precision multilayers ellipsometry and in sensing applications.
\end{abstract}

Keywords: dielectric metamaterial, multilayer, effective medium, photonic crystal, nanostructured device.

\section{Introduction}

Multilayer optics - the study of light propagation in photonic multilayer structures - is the cornerstone subject within the broader field of electrodynamics of inhomogeneous media [1]. On the conceptual level, the multilayer geometry is the simplest possible case of inhomogeneous media: a multilayer structure, being fully homogeneous in two spatial directions and piecewise homogeneous in the third direction, is only one step apart from a truly continuous medium in terms of complexity. On the methodological level, photonic multilayers are subject to several simple and illustrative mathematical theories for their analysis (see, for example, [2] and historical overview in [3]). This concerns both spatially infinite periodic multilayers, which exemplify a very simple variety of one-dimensional photonic crystals [4], and multilayers with a finite number of layers, for which very efficient semi-analytical approaches were developed, based on the transfer matrix [1,2,5] and Airy-type recurrent relations [6,7] formalism. Finally, on the practical level, multilayers easily lend themselves to various planar deposition methods and can, therefore, be fabricated in a reliable and cost-effective manner. For all these reasons, photonic multilayers are one of the most extensively studied optical systems to date, with profound theoretical knowledge and many established applications [8]. As a few characteristic examples, one may mention antireflection coatings, all-dielectric Bragg mirrors and omnidirectional reflectors [9,10], band-pass and multiband-pass filters [2,11,12], devices with tailored 
frequency and group velocity dispersion [13,14], and devices with enhanced nonlinear optical effects $[15,16]$. Moreover, the optical multilayer model can be transferred to other physical systems governed by similar equations, such as acoustic multilayers [1,17] and multiple-quantum-well heterostructures [18].

As was shown in these extensive studies, most interesting properties of photonic multilayers originate from optical interference effects similar to those that underlie the physics of photonic crystals [4]. Therefore, research has traditionally focused on structures with characteristic layer thicknesses $d$ comparable to the wavelength of light $\lambda$. As a notable example, the Bragg mirror, which is none other than a stack of alternating low-and high-index dielectric layers, features maximum reflectance if the optical thickness of each layer is close to one quarter of the wavelength [19]. Structures with much larger layer thicknesses qualify as macroscopic optical systems (also well-studied but with somewhat different methodology). On the other hand, structures with much smaller layer thicknesses $(d \ll \lambda)$ have long been thought of as not very interesting from the photonic band gap structures point of view. Indeed, if the layers are much thinner than the wavelength of light, then the field variations inside such layers should be very small, leading to negligibly weak interference effects. Therefore, it can be adopted that the light wave would not feel the individual layers in such a "deeply subwavelength" structure. Instead, light can be thought of as interacting with the entire composite material, which can be treated as effectively homogeneous and assigned some effective material parameters (see, e.g., [20] and references therein) in much the same way as the ordinary materials are treated as homogeneous media despite having atomic, molecular, or any other intrinsic structure.

This concept of homogenization, which can be traced back to the earliest days of electromagnetic theory in presence of media, becomes really central in metamaterials [21], defined as artificial composite materials where the elements ("meta-atoms") are much smaller than the wavelength. Since the geometry and composition of the meta-atoms can theoretically be arbitrary, the effective medium parameters of metamaterials such as negative refractive index [21-23] or near-zero dielectric permittivity [24] can be very different from those of the naturally occurring materials.

Therefore the applicability of the homogenization concept to particular metamaterials has been a topical and ongoing discussion since the conception of the field [20,25]. While it was found that there are intricate metaatom geometries that do not readily lend themselves to homogenization [25], the applicability of the effective medium theory to the geometries as simple as a multilayer stack have hardly ever been questioned. Even though subwavelength metal-dielectric stacks with effectively indefinite dielectric permittivity tensor (planar hyperbolic metamaterials [26,27]) were found to be subject to a much tighter subwavelength condition than simply $d \ll \lambda[28]$, the origin of this tighter restriction on $d$ results from the existence of plasmonic propagating waves with wave vector $k$ significantly larger than the one inferred from the vacuum wavelength $\left(k_{0}=c / \omega\right)$, and consequently, with actual wavelength $\lambda$ much smaller than $\lambda_{0}=2 \pi c / k_{0}$ [2932]. The principal condition of homogenization applicability, namely that the layer thicknesses must be small compared with the wavelength of light that interacts with them, remains unchanged. Hence, all-dielectric subwavelength multilayers, in which no plasmonic waves with extremely large wave vectors are supported, have been expected to obey the effective medium approximation (EMA) very well [33,34].

Nevertheless, a recent theoretical paper by H.H. Sheinfux et al. [35] demonstrates that this commonly believed assumption may fail in certain regimes. Namely, when the angle of incidence is close to the critical angle of the total internal reflection, the actual multilayer and its effective-medium counterpart can have significantly different transmission spectra despite the deeply subwavelength layer thicknesses (smaller than $1 / 50$ of the wavelength). Moreover, it was shown that the spectra become sensitive to variations of the layer 
thicknesses (the structure period variation) on the scale of one nanometer, i.e. 500 times smaller than the wavelength. Additionally, if the refractive indices of the medium behind the multilayer is carefully chosen, the transmission spectra become dependent on the choice of the outermost ("opening") layer (i.e., $\mathrm{ABAB} \ldots \mathrm{AB}$ vs. BABA...BA), totally contrary to the effective medium theory results. This "anomalous" EMA breakdown reportedly happens because for a wave incident at a near-critical angle, the optical properties of the multilayer arise from the interference effects between phases of Fresnel reflection and transmission coefficients in the frustrated total internal reflection regime rather than between phases accumulated during wave propagation in subwavelength layers [35].

Such EMA breakdown is both enlightening and practically promising, e.g. for sensing and switching applications. However, the breakdown of the EMA reported in [35] only becomes noticeable when the multilayer contains a large number of layers (on the order of several hundreds), which should have equal thickness, as even a very minor thickness randomness would smear the effect due to its extreme sensitivity to the layer thicknesses. Such a structure, having at least 150-200 layers with $10 \mathrm{~nm}$ thickness and $<1 \mathrm{~nm}$ tolerance, would be impractical for any experimental realization. To facilitate the experimental verification and practical applications of this newly observed EMA breakdown, it is necessary to reproduce this effect in practically realizable structures.

In this paper we present a systematic analysis of the anomalous EMA breakdown in deeply subwavelength all-dielectric multilayers. We start from the dispersion relation discrepancies in infinite and semi-infinite multilayers and move on to the description of the effect in finite-thickness multilayer slabs. We then suggest a resonator-enabled geometry, where the EMA breakdown is further enhanced. We identify the parameter range, where the EMA breakdown is the strongest and can be observed in structures feasible for fabrication using state-of-the-art techniques such as atomic layer deposition (ALD). Specifically, we demonstrate that the difference between the reflectance of actual multilayer and the EMA prediction can be as large as 0.98, and the difference between reflectance for different opening layers (ABAB...AB vs. BABA...BA) can reach 0.99. These results can be employed to improve the accuracy and reliability of ellipsometric measurements, to devise new high-precision ellipsometry techniques, and to design new types of ultracompact all-dielectric refractive index sensors with much lower losses compared to plasmonics-based ones.

The remainder of the paper is organized as follows. We describe the methodology of our study in the Section 2. Section 3 follows with the results, with subsections covering different geometries: infinite, semi-infinite, finite multilayer slab, and finite slab with added resonator layer. Finally, in Section 4 we discuss the results and conclude. 


\section{Methods}

We consider the multilayer structure shown in Figure 1(a), consisting of two lossless dielectrics with refractive indices $n_{A}$ and $n_{B}$ and thicknesses $d_{A}$ and $d_{B}$, respectively. The total period is $d=d_{A}+d_{B}$, and we define the filling fraction of material A as $f=d_{A} /\left(d_{A}+d_{B}\right)$. Since the theoretical study presented here neglects material dispersion for the sake of simplicity, it is convenient to normalize all geometrical sizes to the free-space wavelength of incident light $\lambda$, which is what we do throughout the paper.

Conventional Maxwell-Garnett homogenization applied to the multilayer geometry does not introduce any approximations other than the assumption that the layer thicknesses are much smaller than the wavelength. It gives the effective permittivity tensor [5] $\hat{\varepsilon}=\operatorname{diag}\left(\varepsilon_{t}, \varepsilon_{t}, \varepsilon_{z}\right)$, where

$$
\begin{aligned}
\varepsilon_{t} & =f \varepsilon_{A}+(1-f) \varepsilon_{B}, \\
\varepsilon_{z} & =\left(\frac{f}{\varepsilon_{A}}+\frac{1-f}{\varepsilon_{B}}\right)^{-1},
\end{aligned}
$$

and the coordinate system used is given in Figure 1(a). The dispersion equation for the waves in such an ideal homogenous uniaxial medium yields circular isofrequency contours for one polarization (TE or transverse electric with $E_{z}=0$ ) and elliptical isofrequency contours for the other polarization (TM or transverse magnetic with $H_{Z}=0$ ):

$$
\begin{aligned}
& q^{2}+\kappa^{2}=\varepsilon_{t}(T E), \\
& \frac{q^{2}}{\varepsilon_{z}}+\frac{\kappa^{2}}{\varepsilon_{t}}=1(T M),
\end{aligned}
$$

where $q=k_{z} / k_{0}$ and $\kappa=k_{t} / k_{0}$ are normalised wavevector components and $k_{0}=\frac{\omega}{c}=\frac{2 \pi}{\lambda}$ is the wavevector in vacuum.

(a)

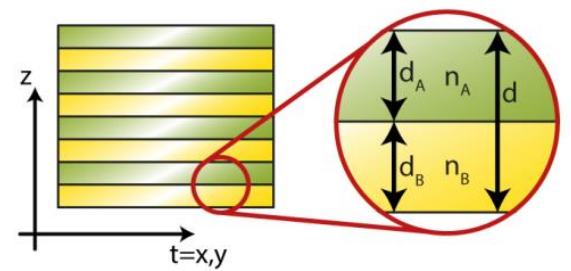

(d)

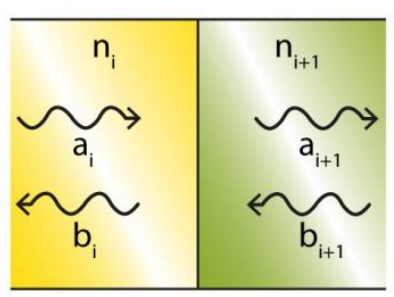

(b)

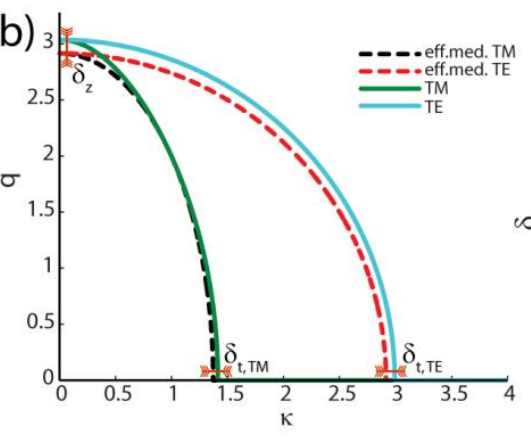

(e)

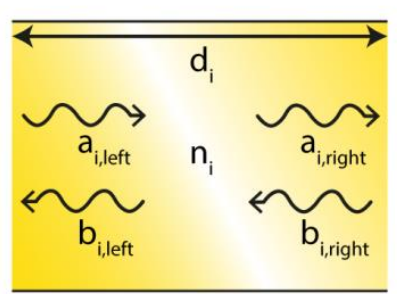

(c)

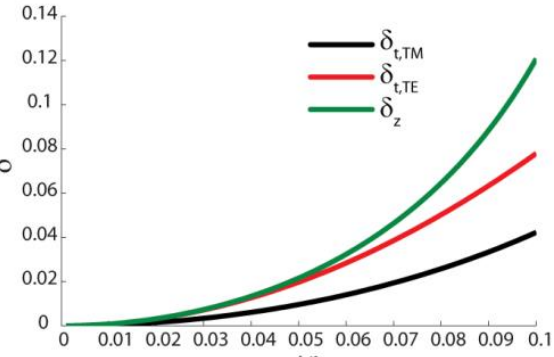

$\mathrm{d} / \lambda$

(f)

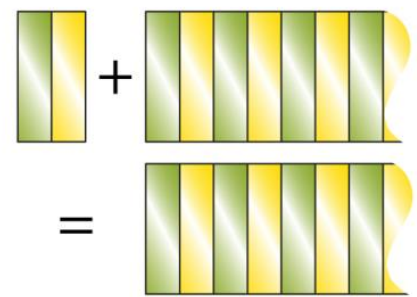

Figure 1. (a) Schematics of the multilayer dielectric structure considered. (b) Example of the isofrequency contours for the homogenized uniaxial medium $\left(n_{A}=1, n_{B}=4, f=0.5, d / \lambda=0.1\right)$ according to Eqs. (1)-(2) (TM - dashed black, TE - dashed red) and solutions of dispersion equation (3) (TM-solid green, TE - solid cyan). (c) Discrepancy between the periodic material and homogenised medium increases with period $d\left(n_{A}=1, n_{B}=4, f=0.5\right)$. (d-f) Illustration of the transfer matrix method: (d) an interface between two dielectrics, (e) propagation in a homogenous layer, (f) illustration of the main idea behind the analytic expression (7) for Fresnel reflection from a semi-infinite structure. 
The dispersion relation for the actual multilayer structure can also be determined analytically. It is known from Bloch's theorem that the existence condition for propagating waves in a one-dimensional periodic medium is directly related to the properties of one period of the structure and the dispersion equation is [28]

$$
\cos \left(q k_{0} d\right)=\cos q_{A} k_{0} d_{A} \cos q_{B} k_{0} d_{B}-\frac{1}{2}\left(K+\frac{1}{K}\right) \sin q_{A} k_{0} d_{A} \sin q_{B} k_{0} d_{B},
$$

where $q_{A, B}=\sqrt{n_{A, B}^{2}-\kappa^{2}}$ and $K=q_{2} / q_{1}$ (TE) or $K=\left(\varepsilon_{1} / \varepsilon_{2}\right)\left(q_{2} / q_{1}\right)$ (TM).

Typically, the isofrequency contour from Eq. (3) for the TE polarization results in an ellipse of a larger size than homogenization predicts [Figure 1(b)], while the contour for the TM polarization is of a more complex shape than elliptical. For a certain value of tangential component $\kappa$ equations (2) and (3) give the same $q$ for the TM polarization.

To characterize the discrepancy between the Bloch and homogenized solutions, we introduce the quantities [see Figure 1(b)]

$$
\delta_{z}=\left.\left(q_{\text {Bloch }}-q_{\text {homogenized }}\right)\right|_{\kappa=0}, \quad \delta_{t}=\left.\left(\kappa_{\text {Bloch }}-\kappa_{\text {homogenized }}\right)\right|_{q=0},
$$

These quantities are chosen because the discrepancy between the homogenized and the Bloch dispersion relations are maximum for $\kappa=0$ and $q=0$, as seen in Figure 1(b). Both these quantities increase with the structure period, as shown in Figure 1(c). The dependency is very close to quadratic, and for smaller period values $d / \lambda<0.05$ it was confirmed that $\delta_{z, t} \sim(d / \lambda)^{2}$.

For calculations of the reflectance from finite-sized multilayer structures we used the transfer matrix method similar to [2], which we briefly outline here for the sake of completeness. For an interface between two materials [see Figure 1(d)] with refractive indices $n_{i}$ and $n_{i+1}$ the relations between electric field amplitudes $a$ and $b$ corresponding to the waves propagating in the two directions are

$$
\left(\begin{array}{l}
a_{i} \\
b_{i}
\end{array}\right)=M_{i, i+1}\left(\begin{array}{l}
a_{i+1} \\
b_{i+1}
\end{array}\right)=\frac{1}{s_{21}}\left(\begin{array}{cc}
1 & s_{11} \\
s_{11} & 1
\end{array}\right)\left(\begin{array}{l}
a_{i+1} \\
b_{i+1}
\end{array}\right),
$$

where $s_{11}=\frac{1-\gamma}{1+\gamma}, s_{21}=\frac{2 \gamma}{1+\gamma}$ and $\gamma=\frac{q_{i+1}}{q_{i}}$ (TE) or $\gamma=\frac{q_{i+1}}{q_{i}} \frac{\varepsilon_{i}}{\varepsilon_{i+1}}=\frac{q_{i+1}}{q_{i}} \frac{n_{i}^{2}}{n_{i+1}^{2}}(\mathrm{TM})$.

For propagation inside layer $i$ [see Figure 1(e)]

$$
\left(\begin{array}{l}
a_{i, \text { left }} \\
b_{i, \text { left }}
\end{array}\right)=P_{i}\left(\begin{array}{l}
a_{i, \text { right }} \\
b_{i, \text { right }}
\end{array}\right)=\left(\begin{array}{cc}
\exp \left(-i q_{i} k_{0} d_{i}\right) & 0 \\
0 & \exp \left(i q_{i} k_{0} d_{i}\right)
\end{array}\right)\left(\begin{array}{l}
a_{i, \text { right }} \\
b_{i, \text { right }}
\end{array}\right) .
$$

The transfer matrix $M$ for an arbitrary number of layers $2 N$ is obtained through subsequent multiplication of matrices $M=\left(\begin{array}{ll}m_{11} & m_{12} \\ m_{21} & m_{22}\end{array}\right)=M_{\mathrm{in}, 1} P_{1} M_{1,2} \ldots M_{2 N-1,2 N} P_{2 N} M_{2 N, \text { out }}$, where the subscripts "in" and "out" denote the ambient media to the both sides of the stack. The reflectance from the structure is then $R=|r|^{2}=$ $\left|\frac{m_{21}}{m_{11}}\right|^{2}$. For example, the transfer matrix $M_{P}$ for one period of the considered structure (dielectric layers A and B are surrounded with material $n_{\mathrm{in}}$ from both sides) is $M_{P}=\left(\begin{array}{ll}m_{P 11} & m_{P 12} \\ m_{P 21} & m_{P 22}\end{array}\right)=M_{\mathrm{in}, A} P_{A} M_{A, B} P_{B} M_{B, \mathrm{in}}$. The transfer matrix for $N$ periods of such a structure can then be written as $\left(M_{P}\right)^{N}$, using the identity $M_{B, \mathrm{in}} M_{\mathrm{in}, A}=M_{B, A}$. 
Finally, to calculate the reflectance from a semi-infinite stack we use the fact that adding one period to a semi-infinite structure does not change the overall reflectance, as illustrated in Figure 1(f). Thus

$$
\left(\begin{array}{c}
1 \\
r_{\infty}
\end{array}\right)=M_{P}\left(\begin{array}{c}
1 \\
r_{\infty}
\end{array}\right) F=\left(\begin{array}{ll}
m_{P 11} & m_{P 12} \\
m_{P 21} & m_{P 22}
\end{array}\right)\left(\begin{array}{c}
1 \\
r_{\infty}
\end{array}\right) F,
$$

where the factor $F$ stands for the reduction of the wave amplitude after propagation through one period. Equation (7) gives a quadratic equation for determination of $r_{\infty}$ with two roots. One of the roots, for which the reflectance is larger than 1 , is discarded as non-physical. 


\section{Results}

\subsection{Infinite multilayer structure: dispersion equation}

We start our investigation from the infinite periodic multilayer structure [Figure 1(a)]. In such a system, there are four independent parameters to vary: refractive indices $n_{A}, n_{B}$ and layer thicknesses $d_{A}, d_{B}$ expressed as the lattice period $d=d_{A}+d_{B}$ and the filling fraction $f=d_{A} / d$. Most of the low-loss dielectrics in the optical and near infrared region have refractive indices in the range $1-4$, therefore we stay within this limit.

Figures 2 and 3 show the characteristic dependencies of the discrepancies $\delta_{t, T M}, \delta_{t, T E}$ and $\delta_{z}$, as defined in Equation (4), on refractive indices. We can see that the discrepancies increase with the difference of refractive indices $\left|n_{A}-n_{B}\right|$ nearly monotonically (see Figure 2). This gradual decrease of the accuracy of EMA is the expected result since the optical thickness of a layer increases with its index of refraction, so even for a physically subwavelength layer (e.g., $d_{B} / \lambda=0.05$ ), one can have $n_{B} d_{B} / \lambda=0.2$ for $n_{B}=4$, which is already approaching the quarter-wave layer optical thickness of 0.25 . We note that the dependence of $\delta_{t, T M}$ on $n_{A}$ and $n_{B}$ is slightly more complicated than for $\delta_{t, T E}$ and $\delta_{z}$, namely, the isolines $\delta_{t, T M}\left(n_{A}, n_{B}\right)=$ const are slightly curved rather than straight [cf. Figure 2(a) and (b-c)]. This means that, e.g., the dependence $\delta_{t, T M}\left(n_{A}=\right.$ const, $\left.n_{B}\right)$ has a maximum for $1<n_{B}<4$, whereas the corresponding functions $\delta_{t, T E}\left(n_{A}=\right.$ const, $\left.n_{B}\right)$ and $\delta_{Z}\left(n_{A}=\right.$ const, $\left.n_{B}\right)$ are monotonic in that range.
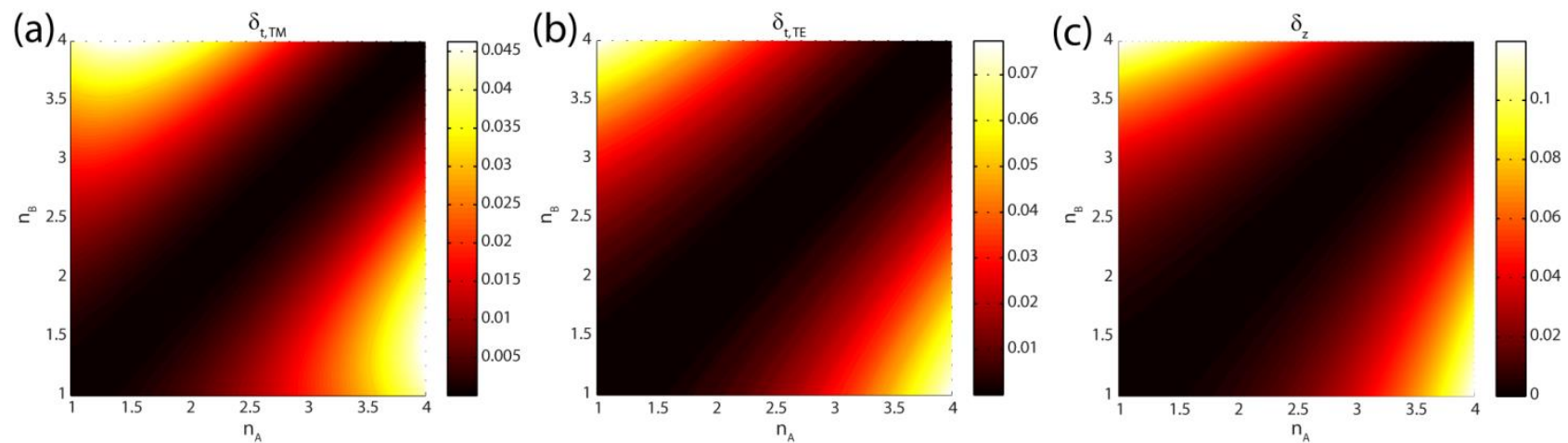

Figure 2. Discrepancies $\delta_{t, T M}(\mathrm{a}), \delta_{t, T E}(\mathrm{~b})$ and $\delta_{z}$ (c) between Bloch and EMA dispersion equation increase with the difference of refractive indices $\left|n_{A}-n_{B}\right|$. Other parameters are $d / \lambda=0.1$ and $f=0.5$.

The same gradual frustration of EMA can be observed in the monotonically increasing dependencies of $\delta_{t, T M}, \delta_{t, T E}$ and $\delta_{z}$ on the lattice period $d$, as shown in Figure 3. To be able to consider the refractive indices of surrounding materials below and above $n_{A}$ and $n_{B}$ while staying within the range $1-4$ for all materials, we fix $n_{A}=2$ and $n_{B}=3$ and use them throughout the rest of the paper. The dependencies of $\delta_{t, T M}, \delta_{t, T E}$ and $\delta_{z}$ on filling fraction $f$ feature a maximum at values between 0.3 and 0.6 depending on polarization. This is also reasonable, since for the limiting cases $f=0$ and $f=1$ the multilayer stack becomes a homogenous material for which the EMA is automatically valid; on the other hand, at the intermediate values of $f$ the effects brought about by the non-homogeneity of the structure are expected to be stronger.

Since the dependencies in Figures 2 and 3 are featureless and only predict the gradual frustration of the EMA as the layers become optically thicker, we can pick any values of multilayer parameters for further investigation in other geometries. For the rest of the paper we use the period size $d / \lambda=0.02$. For this period the maximum of $\delta_{t, T M}$ occurs for $f=0.35$, maximal $\delta_{t, T E}$ and $\delta_{z}$ are reached for $f=0.52$, and for 
the filling fraction $f=0.47$ all $\delta$ are equal [Figure 3(d)]. To facilitate direct comparison between the two polarizations, we stick to this filling fraction $f=0.47$ throughout the rest of the paper, yielding the permittivity tensor components $\varepsilon_{t}=2.579^{2}$ and $\varepsilon_{z}=2.381^{2}$ according to Equations (1).

(a)

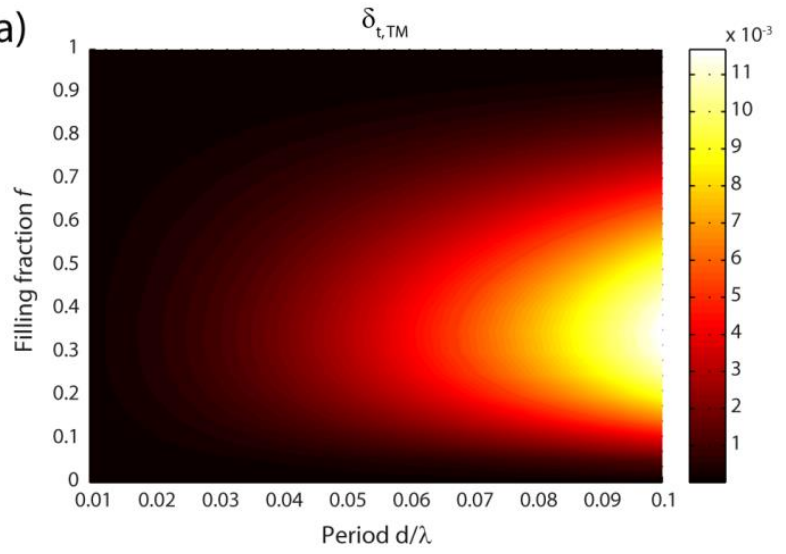

(c)

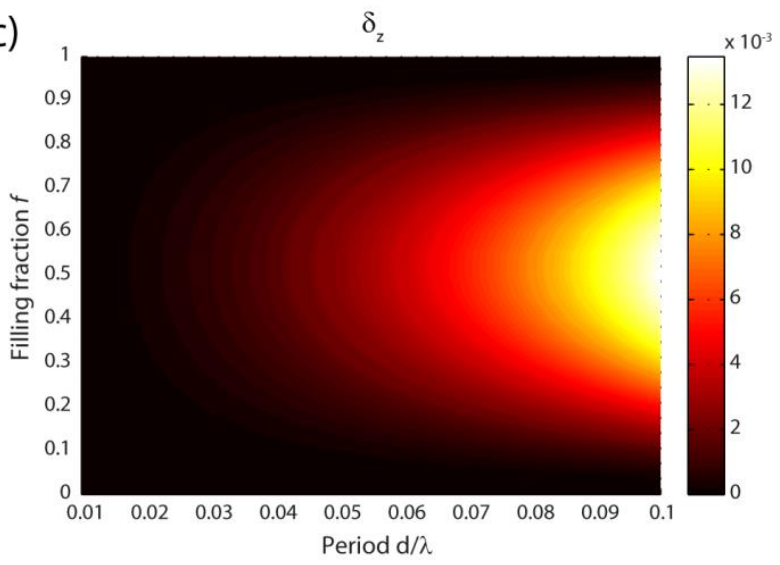

(b)

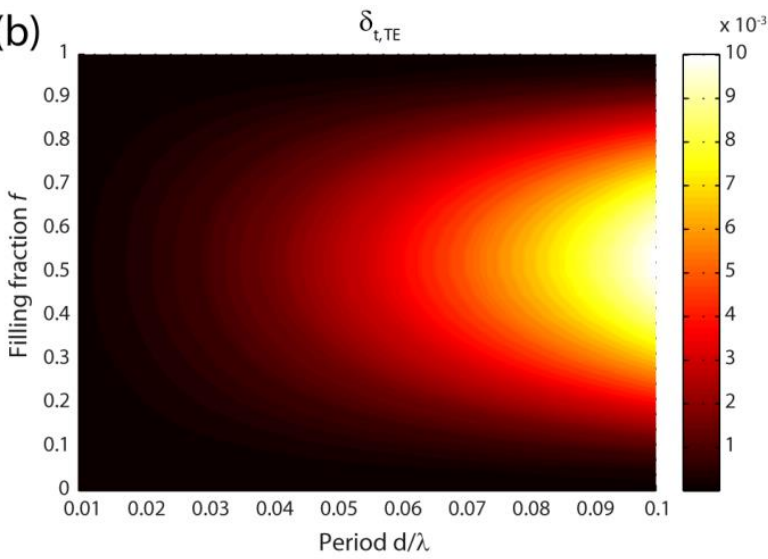

(d)

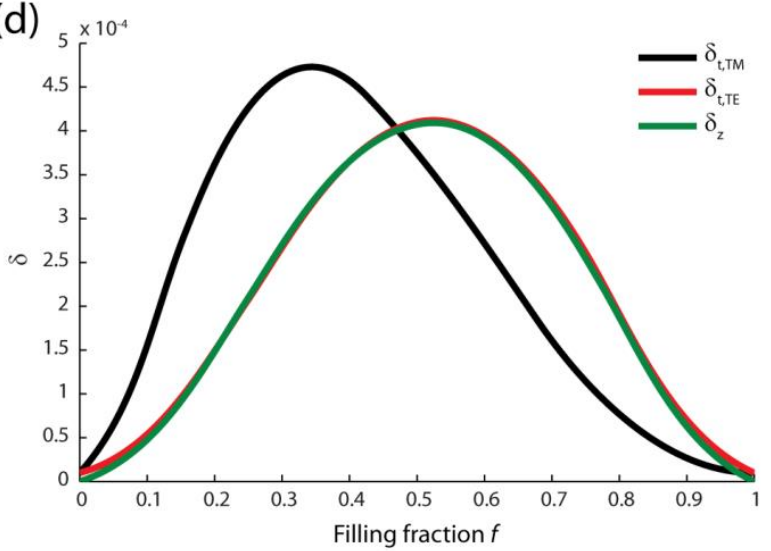

Figure 3. (a-c) Discrepancies $\delta_{t, T M}(\mathrm{a}), \delta_{t, T E}$ (b) and $\delta_{z}$ (c) between Bloch and EMA dispersion equation increase with the period $d / \lambda$ and experience maximum at a certain filling fraction $f$ for a fixed $d / \lambda$. Refractive indices are $n_{A}=2$, $n_{B}=3$. (d) Discrepancies for $d / \lambda=0.02$ experience maximum at the filling fraction $f=0.35\left(\delta_{t, T M}\right)$ and $f=0.52$ $\left(\delta_{t, T E}\right.$ and $\left.\delta_{z}\right)$; they are equal for the crossing point at $f=0.47$.

\subsection{Semi-infinite multilayer structure}

Our next goal is to consider a structure that would be minimally more complex than an infinite periodic multilayer, i.e., a semi-infinite periodic multilayer bordering some ambient homogeneous material with refractive index $n_{\mathrm{in}}$. Since the translation invariance is broken by that interface, the Bloch theorem is no longer rigorously applicable, so in order to characterize the EMA applicability, we analyze discrepancies in the Fresnel reflection coefficient for a wave incident from the ambient medium onto the multilayer structure. Such scenario is also much more relevant than the dispersion relation from the experimental point of view because the reflectance is directly measurable.

Specifically, and following two main results in [35], we will introduce two quantities:

$$
\begin{aligned}
& \Delta R_{d}=R_{d / \lambda=0.02}-R_{d / \lambda=0.001} \\
& \Delta R_{o}=\left.\left(R_{\mathrm{ABAB} \ldots}-R_{\mathrm{BABA} \ldots}\right)\right|_{d / \lambda=0.02} .
\end{aligned}
$$


The first one, $\Delta R_{d}$, is essentially the difference between the reflectance of the actual multilayer and its homogenized counterpart, since the structure with $d / \lambda=0.001$ can be regarded as "quasi-homogenous"; we recall that all discrepancies $\delta$, as defined in Equation (4), scale quadratically with the period of the structure [cf. Figure 1(c)], so $d / \lambda=0.001$ is " 400 times more homogenous" than $d / \lambda=0.02$. This quantity will be non-zero if finite thickness of layers, even as small as $\lambda / 50$, has an influence on the reflectance of the entire multilayer, indicating the breakdown of the EMA. The second quantity in Equation (8), namely $\Delta R_{o}$, is the difference between reflectance for the same structure (with $d / \lambda=0.02$ ) starting with the either layer A or layer B. This quantity signifies the influence of the layers ordering (hence the subscript " $o$ "), again indicating the breakdown of homogenization because, obviously, two otherwise identical multilayers that only differ by layer ordering are homogenized to the same material.

In addition to the parameters of the multilayer stack analysed in the previous section, there are two additional parameters, namely the above-mentioned refractive index of the ambient material $n_{\text {in }}$ and angle of incidence $\alpha$ for the impinging wave. It was found that layer ordering difference $\Delta R_{o}$ is negligible (on the level of the numerical noise), while layer thickness difference $\Delta R_{d}$ is shown in Figure 4. It can be seen in Figure 4 (a) and (b), where the logarithmic scale is used for clarity, that $\Delta R_{d}$ is small (around $10^{-3}$ ) for most $n_{\text {in }}$ and $\alpha$ below the critical angle. Interestingly there are distinct lines of local minima [two crossing lines for TM in Figure 4(a) and one line for TE in Figure 4(b)], corresponding to crossings between the homogenized and actual multilayer dispersion relations, as seen in Figure 1(b). In contrast, for $\alpha$ above the critical angle (where total internal reflection occurs) the reflectance difference is exactly zero [black areas in Figure 4 (a) and (b)], making the transition to the total internal reflection regime clearly marked.

The most significant feature of the dependence $\Delta R_{d}\left(n_{\mathrm{in}}, \alpha\right)$ is seen to occur immediately before the critical angle. As seen in Figure 4(c) and (d), where the region in the vicinity of the critical angle is magnified, and as confirmed by plotting the explicit reflectances in Figure 4(e)-(f), the reflectance difference can be as large as 0.1 . This occurs for the combination of parameters when the homogenized multilayer is in the total internal reflection regime, so that $R_{d / \lambda=0.001}=1$, but the field in multilayer with finite layer thicknesses, weakly evanescent in lower-index (A) layers and propagating in higher-index (B) layers, forms a quasipropagating Bloch wave (giving $R_{d / \lambda=0.02}<1$ ). This regime corresponds to the case described in [35], and therefore Figure 4 confirms that the anomalous EMA breakdown actually occurs at near-critical incident angles and can be present already in semi-infinite multilayers.

In what follows, we will focus on the detailed investigation of this anomalous breakdown in a variety of geometries, and will therefore use the input refractive index $n_{\text {in }}=3$ for the rest of the paper. This value corresponds to the critical angle 52.5 degrees (TM) and 59.3 degrees (TE) for the quasi-homogenous structure. Figure 4 (e) and (f) shows the reflectance from the actual and quasi-homogenous structures for this value. It is seen that the difference $\Delta R_{d}$ is maximal at the incidence angle exactly equal to the critical angle. Therefore we will use $\alpha=52.5^{\circ}$ (TM) and $\alpha=59.3^{\circ}$ (TE) for the rest of the paper. 
(a)

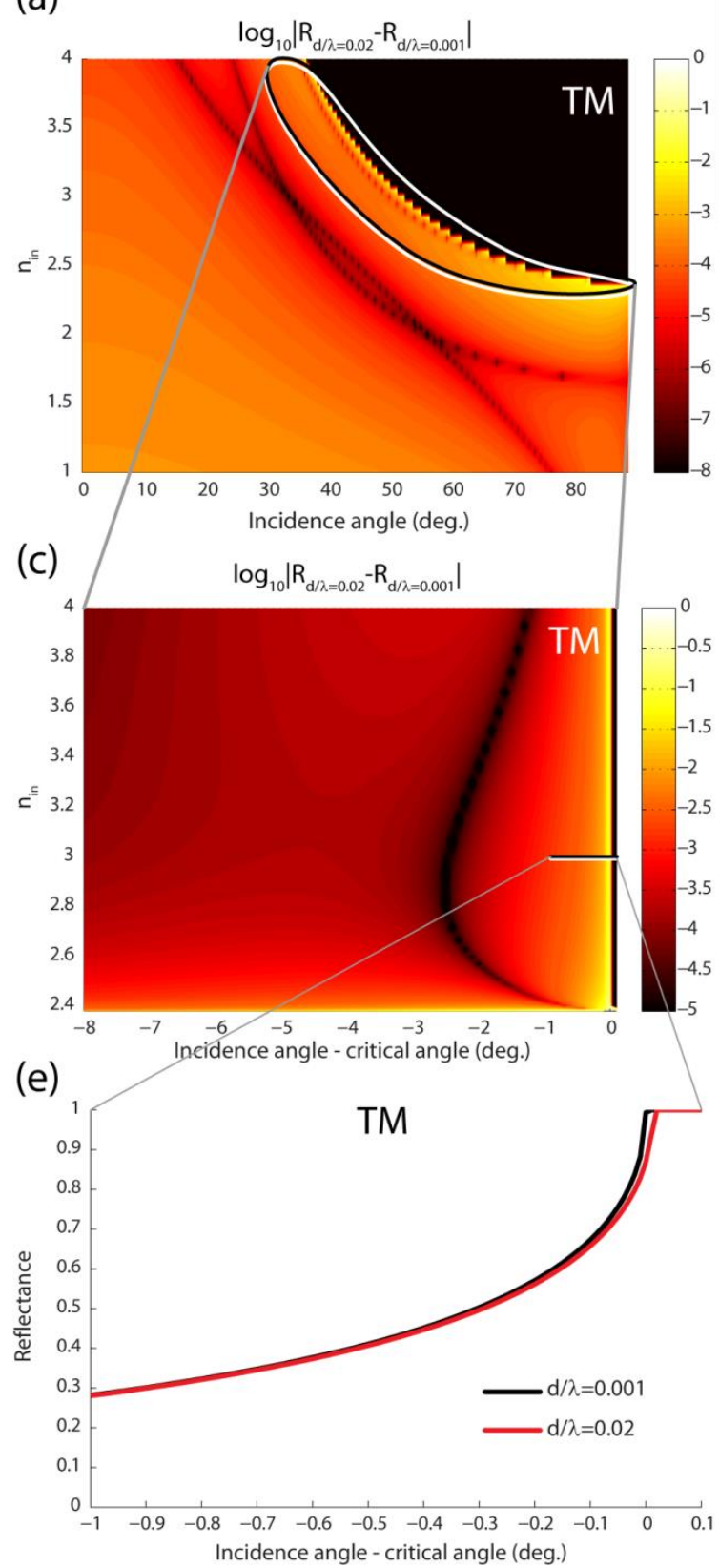

(b)

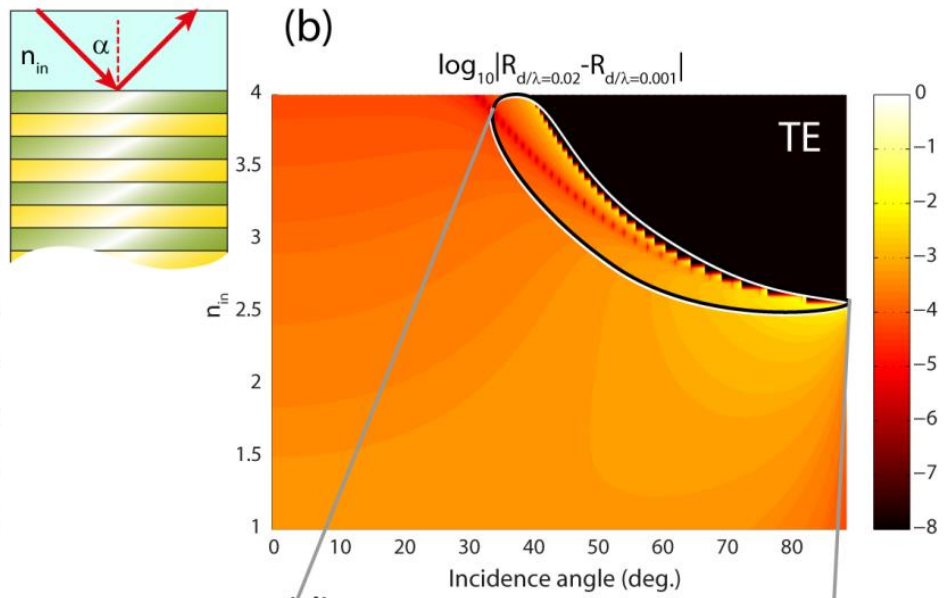

(d)
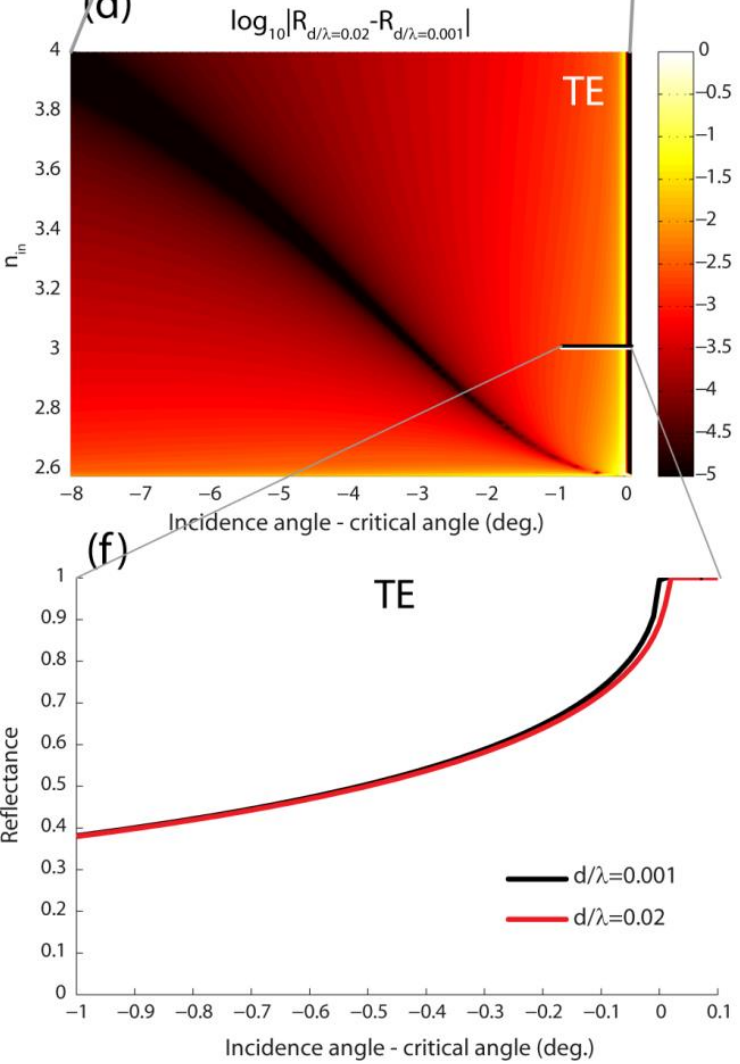

Figure 4. (a-b) Difference of reflectance $\left|R_{d}\right|=\left|R_{d / \lambda=0.02}-R_{d / \lambda=0.001}\right|$ for the actual periodic structure and quasihomogenous structure. Reflectance difference in a broad range of input refractive indices $n_{\text {in }}$ and incidence angles $\alpha$ for TM (a) and TE (b) polarizations. Black area corresponds to the total internal reflection. (c-d) Region of $n_{\text {in }}$ and $\alpha$ close to the critical (total internal reflection) angle for TM (c) and TE (d). (e-f) Reflectances for the periodic $(d / \lambda=0.02)$ and quasi-homogenous $(d / \lambda=0.001)$ structures are significantly different only in the vicinity of the critical angle $\left(n_{\text {in }}=3\right)$ both for TM (e) and TE (f) polarizations.

\subsection{Finite multilayer stack}

Now we consider the case of a finite multilayer stack, which is the simplest practically relevant geometry because such a stack can be not only characterized in reflectance measurements (as the semi-infinite stack in principle can), but also fabricated. Having already fixed the parameters of the multilayer structure, the ambient material and incidence angle, we vary the parameters specific to this new geometry, namely the 
thickness of the multilayer $D$ and refractive index of the output material $n_{\text {out }}$ (see inset in Figure 5). For the sake of simplicity, we use $D$ as a continuously varying parameter, keeping in mind that all real calculations are made with an integer number of periods.

(a)

(c)

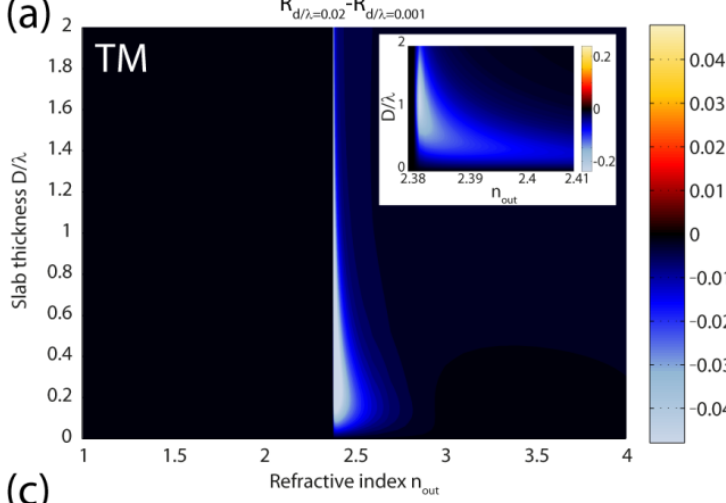

$R_{A}-R_{B}$

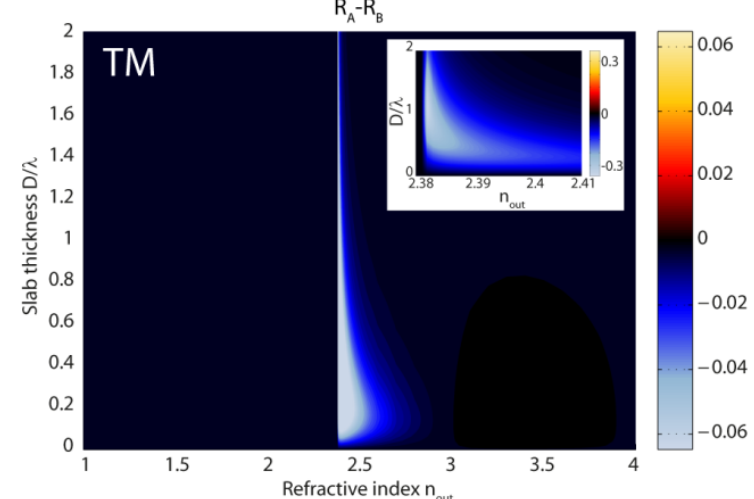

(e)

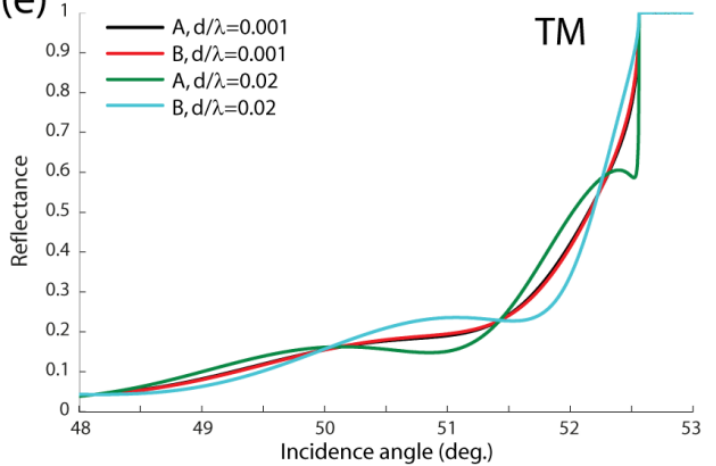

(b)

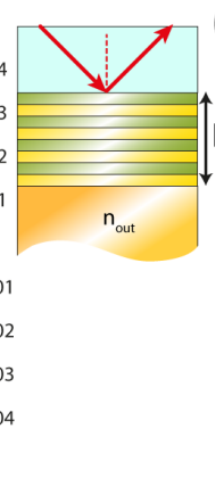

(d)

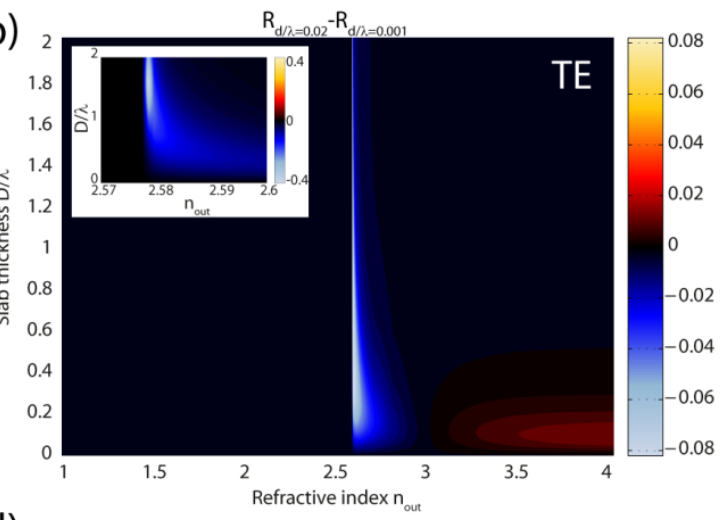

$R_{A}-R_{B}$

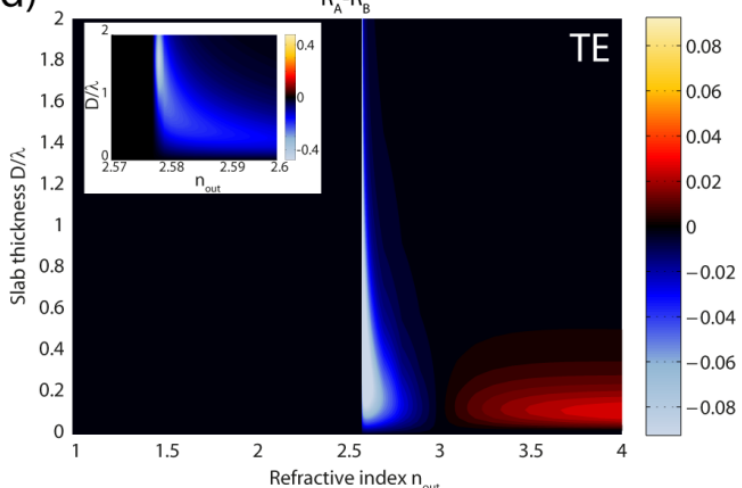

(f)

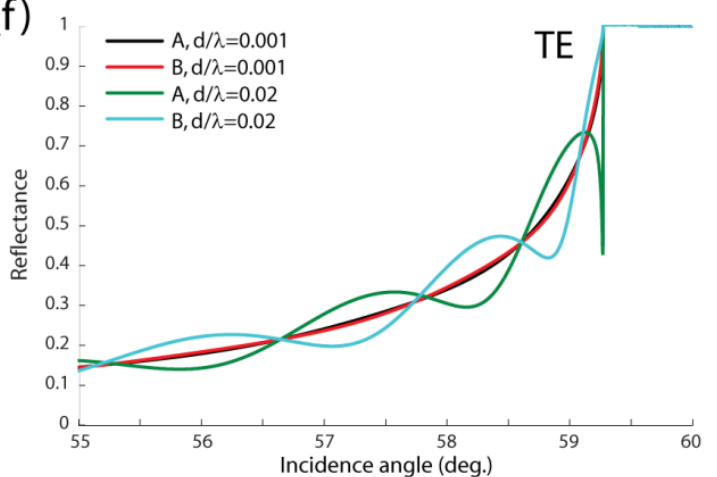

Figure 5. (a-b) Reflectance difference between the actual periodic and quasi-homogenous structures $\Delta R_{d}$ for TM (a) and TE (b) polarizations for the finite stack. (c-d) Reflectance difference $\Delta R_{o}$ for the periodic structure with opening layer A and B for TM (c) and TE (d) polarizations. Insets show the magnified region around maximum reflectance difference. (e-f) Reflectances for A and B opening layer, for periodic and quasi-homogenous structures depending on the angle in the vicinity of the critical angle for TM (e) and TE (f) polarizations.

The resulting dependencies $\Delta R_{d}\left(D, n_{\text {out }}\right)$ and $\Delta R_{o}\left(D, n_{\text {out }}\right)$ are shown in Figure 5. Several interesting features can be seen. First, for the semi-infinite stack $\Delta R_{d}$ can reach values on the order of 0.1 [Figure 4 (a)(b)], while for a finite slab [Figure 5 (a) and (b)] $\Delta R_{d}$ yields the maximum values of 0.24 and 0.41 for TM and TE polarization, respectively. This means that the anomalous EMA breakdown near the critical angle identified in the semi-infinite case persists in the finite multilayer case too. Second, the area where $\Delta R_{d}$ is significantly non-zero is quite narrow along the horizontal axis $n_{\text {out }}$, so the EMA breakdown effect is quite 
sensitive to output refractive index $n_{\text {out }}$. Specifically, the maximum discrepancy is observed for $n_{\text {out }}=$ 2.579 (TE) and $n_{\text {out }}=2.382$ (TM), which is very close to $\sqrt{\varepsilon_{t}}$ and $\sqrt{\varepsilon_{z}}$, respectively. This is in full accordance with the findings in [35], where this high sensitivity was attributed to the impedance matching conditions at the output interface of the multilayers. Third, it is seen that the dependence on $D$ is nonmonotonic as well, but is much weaker. There are rather broad maxima at $D / \lambda=1.64$ (TE) and $D / \lambda=$ 1.12 (TM), and it can be seen that the range of $D / \lambda$ where $\Delta R_{d}$ stays above 0.04 spans from 0.1 to around 2 .

In stark contrast to the semi-infinite multilayers, the ordering reflectance difference $\Delta R_{o}=R_{A}-R_{B}$ [Figure 5 (c) and (d) and insets therein] is significantly greater than zero. It shows the same dependence on $n_{\text {out }}$ and $D$ as $\Delta R_{d}$ reaching the maximal absolute values 0.46 (TE) and 0.32 (TM) at the same values of $n_{\text {out }}$ and $D /$ $\lambda$. Remarkably, the maxima of $\Delta R_{d}$ and $\Delta R_{o}$ are broad with respect to $D / \lambda$, so even though the maxima of the reflectance difference $\Delta R_{d}$ and $\Delta R_{o}$ occur at relatively large multilayer stack thickness $D / \lambda>1$, the reflectance difference is measurable $\left(\Delta R_{d}=0.04\right.$ and $\Delta R_{o}=0.07$ for both TE and TM polarizations) even at the stack thickness as small as $D / \lambda=0.1$ (only five periods).

Figure 5 (e) and (f) show the explicit reflectance dependence on the incidence angle near the critical angle for the multilayered $(d / \lambda=0.02)$ and quasi-homogeneous $(d / \lambda=0.001)$ structures with A or B opening layers. The reflectances for the actual multilayer structure generally follow the quasi-homogenous trend, oscillating around it; oscillations for opening layers A and B are of the opposite signs with respect to the quasi-homogenous line, and all the curves coincide at certain angles. Interestingly, the critical angle shift [observed in Figure 4(e) and (f)] is not observed anymore in Figure 5 (e) and (f). It results from fact that the total internal reflection depends mainly on the substrate, which is identical for all cases.

\subsection{Finite multilayer stack with resonator layer}

The final geometry that we consider here includes an additional finite-thickness layer between the multilayer structure and the substrate (see inset in Figure 6). This intermediate layer acts as a photonic resonator with resonance conditions determined by its thickness $L$. Such a geometry is highly relevant because previous research has shown that a resonator (even a very primitive one such as a single layer of carefully chosen thickness) can considerably improve impedance matching in many photonic devices, leading to enhancement of various effects such as nanoantennas excitation [36] and metasurface polarization conversion [37]. It is expected that the already measurable discrepancies between the reflectance from the actual multilayer and its homogenized counterpart may be enhanced in the resonator set-up.

Following the same adopted methodology of gradually working from the simpler geometry to the more complicated, we fix the refractive index of the resonator layer to be the optimal $n_{\text {out }}$ determined in the previous section, namely, $n_{\text {out }}=2.382(\mathrm{TM})$ and $n_{\text {out }}=2.579(\mathrm{TE})$, and use resonator thickness $L$ and refractive index of the substrate beneath the resonator $n_{\text {sub }}$ as new variable parameters. The total thickness of the multilayer stack is fixed at $D / \lambda=1.64$ (TE) and $D / \lambda=1.12$ (TM). 


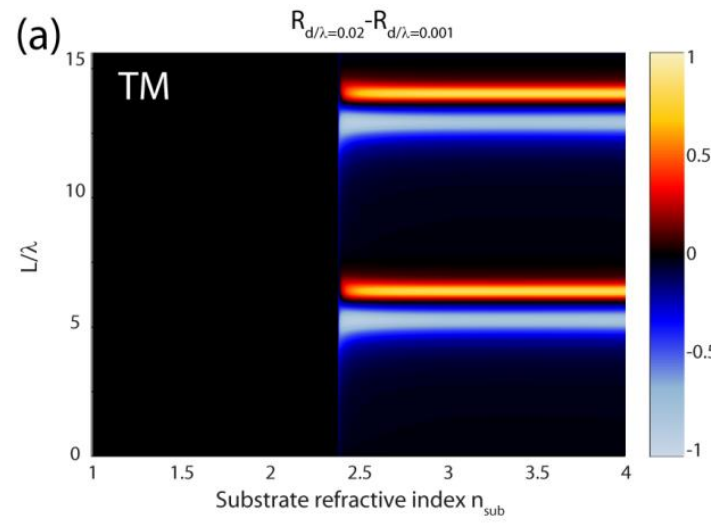

(c)

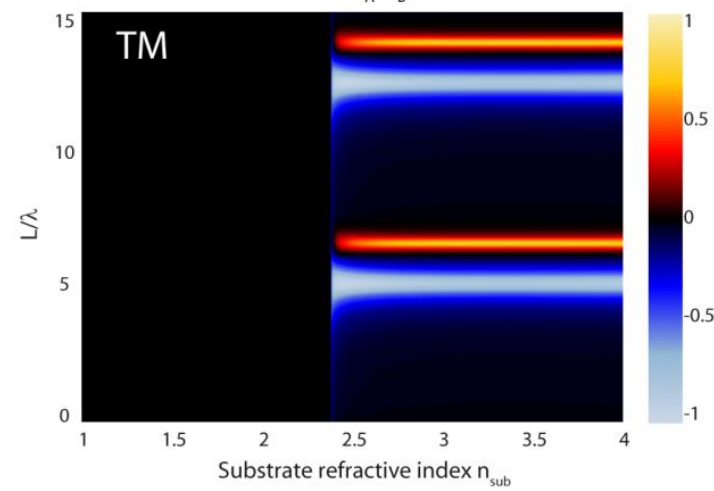

(b)

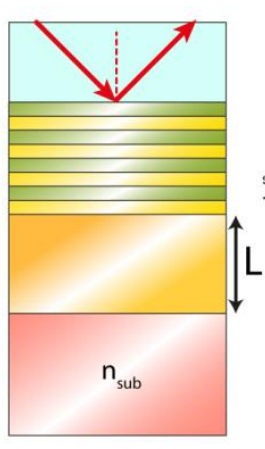

(d)
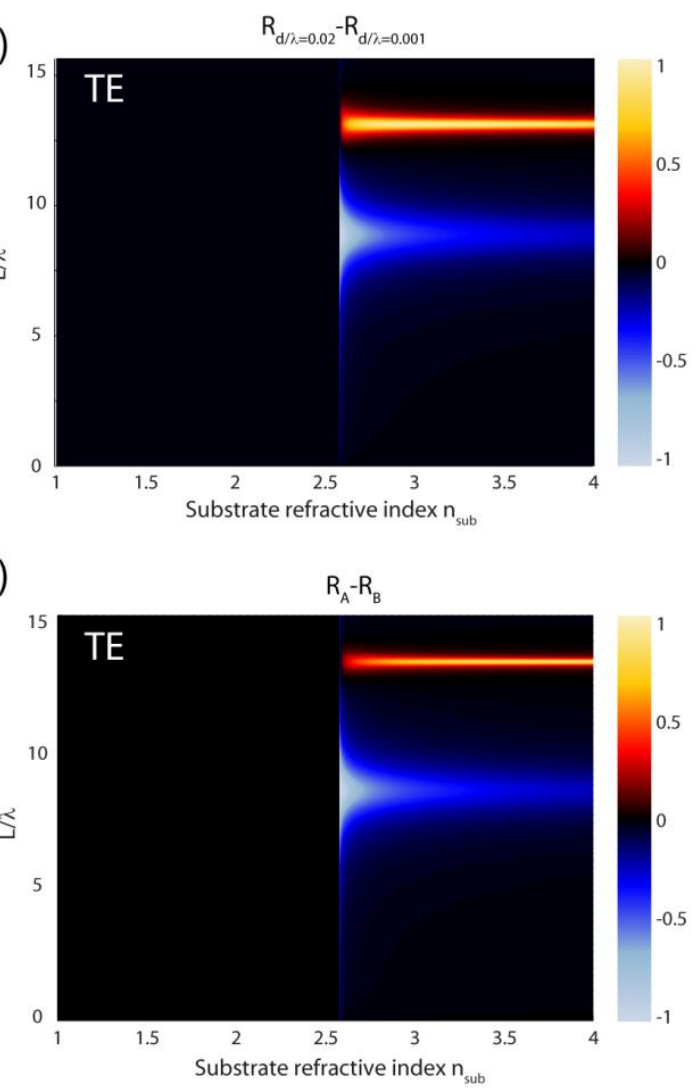

Figure 6. (a-b) Reflectance difference $\Delta R_{d}$ between the actual periodic and quasi-homogenous structures for TM (a) and TE (b) polarizations for the finite stack with an additional layer of refractive index $n_{\text {out }}$ and thickness $L$. (c-d) Reflectance difference $\Delta R_{o}$ for the periodic structure with opening layer A and B for TM (c) and TE (d) polarizations.

The results are shown in Figure 6. As expected from the previous theoretical considerations [37], discrepancies $\Delta R_{d}$ and $\Delta R_{o}$ are greatly enhanced compared to the case when the resonator is absent. The primary factor affecting the enhancement is resonator thickness $L$, with both $\Delta R_{d}$ and $\Delta R_{o}$ periodically exhibiting maxima and minima with changing $L / \lambda$. The dependence on substrate refractive index $n_{\text {sub }}$ is much weaker and essentially consists in the requirement $n_{\text {sub }}>n_{\text {out }}$ : it can be seen in Figure 6 that the EMA breakdown vanishes otherwise. The dependencied $\Delta R_{d, o}\left(n_{\text {sub }}\right)$ achieves saturation once $n_{\text {sub }}$ exceeds $n_{\text {out }}$ substantially, and there is a transition region for $n_{\text {sub }} \gtrsim n_{\text {out }}$ where the resonator has little effect so that the structure beneath the multilayer can be thought of as effectively bulk.

Specifically, finite-thickness difference $\Delta R_{d}$ reaches maximal absolute values 0.90 for $L / \lambda=5.05, n_{\text {sub }}=$ 2.655 (TM) and 0.98 for $L / \lambda=8.50, n_{\text {sub }}=2.587$ (TE) [see Figure 6 (a) and (b)]. Ordering reflectance difference $\Delta R_{o}$ [Figure 6 (c) and (d)] shows qualitatively similar behaviour, reaching maximal absolute values 0.97 for $L / \lambda=5.05, n_{\text {sub }}=2.635(\mathrm{TM})$ and 0.99 for $L / \lambda=8.50, n_{\text {sub }}=2.587$ (TE). For a thinner multilayer stack with $D / \lambda=0.2$ (10 periods), it was shown that the resonator configuration also yields significant maximal reflectance differences, reaching $\Delta R_{d}=0.10$ and $\Delta R_{o}=0.20$ for both TE and TM polarization. 


\section{Discussion and conclusions}

Our results show that all-dielectric subwavelength multilayer stacks exhibit surprisingly interesting properties. Even though their isofrequency contours do not differ much from the effective medium predictions (Figures 2 and 3), it turns out that under certain circumstances (surrounding materials refractive indices, angle of incidence, thickness of the stack) this can be enough to cause significant differences in reflection between the actual structure and EMA prediction (Figures 4-6). These differences clearly manifest as the anomalous breakdown of the EMA applicability. In other words, it is possible that an incident wave can excite guided modes in a multilayer stack, leading to less-than-unity reflectance, but would undergo total internal reflection from a slab of homogeneous material representing the stack under the EMA. Using a finite-size stack (Figure 5) and placing a resonator layer behind the multilayer (Figure 6), it is possible to further enhance the reflectance difference. The reflectance depends not only on the period, but also on the opening layer (A or B) in the stack. In the resonator geometry it is even possible to maximize the reflectance difference (Figure 6) up to 0.99. Our results corroborate the findings of H. Sheinfux et al [35], placing them in context of a systematic and comprehensive investigation and identifying the parameters where these findings could be experimentally verified using existing fabrication and measurement techniques.

The results are even more surprising taking into account that the structure consists of lossless dispersionless dielectrics (contrary to metal-based metamaterials) and no material resonances (contrary to resonant metamaterials) are involved. The analysis presented in this paper is general and wavelength-independent (all the sizes are normalized to the wavelength) so they are applicable for a broad range of frequencies from radio waves to light waves.

Two important practical conclusions can be drawn. First of all, one should be extremely careful doing ellipsometry of multilayer structures, since the applicability of the EMA, usually taken for granted, can be grossly invalid under certain circumstances. Existing ellipsometry models involving multilayer structures may be in need for correction due to this possibility.

Second, we notice that the observed effect generally happens in a narrow parameter window (surrounding materials refractive index, angle of incidence, stack thickness). Hence the investigated anomalous EMA breakdown can be used for sensing applications, where minute variation in the refractive index (primarily that of the medium immediately behind the multilayer structure) can be detected in reflectance measurements. The resonator geometry considered in Section 3.4 is particularly promising because of high performance and because it can be realized with a relatively small number of layers (for example, 10 periods). The multilayer structure can be placed on top of a microfluidic channel for lab-on-a-chip applications. In addition, high sensitivity to the total thickness of the stack, individual layer thickness, and multilayer termination (whether the opening layer is A or B) can be used as a new optical method of in situ monitoring of subwavelength multilayer deposition such as ALD. Finally, high sensitivity to the incidence angle can be used in precise angle measurements and control.

In summary, we have performed a general systematic analysis of deeply subwavelength dielectric multilayers and the anomalous EMA breakdown in such structures. We have determined the conditions for maximal reflectance difference between the actual periodic multilayer and its homogenized counterpart, as well as the maximal sensitivity to the choice of the opening layer in the stack. The presented results will be useful for ellipsometric characterization of multilayer stacks as well as for a variety of sensing applications. 


\section{References}

[1] Brekhovskikh L M 1980 Waves in Layered Media (New York, NY: Academic Press)

[2] Yeh P 1988 Optical waves in layered media (Wiley)

[3] Brillouin L 2003 Wave propagation in periodic structures: electric filters and crystal lattices (Courier Dover Publications)

[4] Joannopoulos J D, Johnson S G, Winn J N and Meade R D 2008 Photonic Crystals: Molding the Flow of Light (Princeton, NJ: Princeton Unuversity Press)

[5] Born M and Wolf E 1999 Principles of Optics (Cambridge: Cambridge University Press)

[6] Mandatori A, Sibilia C, Bertolotti M, Zhukovsky S and Gaponenko S V. 2005 Analytical demonstration of omnidirectional transmission enhancement in dispersive birefringent photonicbandgap structures J. Opt. Soc. Am. B 221785

[7] Zhukovsky S V., Helt L G, Kang D, Abolghasem P, Helmy A S and Sipe J E 2013 Analytical description of photonic waveguides with multilayer claddings: Towards on-chip generation of entangled photons and Bell states Opt. Commun. 301-302 127-40

[8] Macleod H A 2010 Thin-Film Optical Filters, Fourth Edition (Taylor \& Francis)

[9] Winn J N, Fink Y, Fan S and Joannopoulos J D 1998 Omnidirectional reflection from a onedimensional photonic crystal Opt. Lett. 231573

[10] Chigrin D N, Lavrinenko A V, Yarotsky D A and Gaponenko S V 1999 Observation of total omnidirectional reflection from a one-dimensional dielectric lattice Appl. Phys. A Mater. Sci. Process. 68 25-8

[11] Kozhukh S, Zhukovsky S, Yurkevich I, Stepkova S, Gaponenko S, Lunevich A and Glukhov Y 2012 Design of high-transmission multiband multilayer filters for Raman spectroscopy J. Nanophotonics 6 061704

[12] Zhukovsky S V., Gaponenko S V. and Lavrinenko A V. 2014 Optical filters based on fractal and aperiodic multilayers Optics of Aperiodic Structures - Fundamentals and Device Applications ed L Dal Negro (Singapore: Pan Stanford Publishing) pp 91-142

[13] Belardini A, Bosco A, Leahu G, Centini M, Fazio E, Sibilia C, Bertolotti M, Zhukovsky S and Gaponenko S V. 2006 Femtosecond pulses chirping compensation by using one-dimensional compact multiple-defect photonic crystals Appl. Phys. Lett. 89031111

[14] Makarava L, Nazarov M, Ozheredov I, Shkurinov A, Smirnov A and Zhukovsky S 2007 Fibonaccilike photonic structure for femtosecond pulse compression Phys. Rev. E 75036609

[15] D’Aguanno G, Centini M, Scalora M, Sibilia C, Bertolotti M, Bloemer M J and Bowden C M 2002 Generalized coupled-mode theory for $\chi^{\wedge}(2)$ interactions in finite multilayered structures $J$. Opt. Soc. Am. B 192111 
[16] Zhukovsky S V. and Smirnov A G 2011 All-optical diode action in asymmetric nonlinear photonic multilayers with perfect transmission resonances Phys. Rev. A 83023818

[17] Li F and Wang Y 2012 Elastic wave propagation and localization in band gap materials: a review Sci. China Physics, Mech. Astron. 55 1734-46

[18] Zhukovsky S V. and Gaponenko S V. 2008 Constraints on transmission, dispersion, and density of states in dielectric multilayers and stepwise potential barriers with an arbitrary layer arrangement Phys. Rev. E 77046602

[19] Chigrin D N, Lavrinenko A V, Yarotsky D A and Gaponenko S V 1999 All-dielectric onedimensional periodic structures for total omnidirectional reflection and partial spontaneous emission control J. Light. Technol. 17 2018-24

[20] Simovski C R 2011 On electromagnetic characterization and homogenization of nanostructured metamaterials J. Opt. 13013001

[21] Cai W and Shalaev V 2010 Optical Metamaterials (New York, NY: Springer New York)

[22] Zhang S, Fan W, Panoiu N, Malloy K, Osgood R and Brueck S 2005 Experimental Demonstration of Near-Infrared Negative-Index Metamaterials Phys. Rev. Lett. 95137404

[23] Shelby R A, Smith D R and Schultz S 2001 Experimental verification of a negative index of refraction. Science 292 77-9

[24] Maas R, Parsons J, Engheta N and Polman A 2013 Experimental realization of an epsilon-near-zero metamaterial at visible wavelengths Nat. Photonics 7 907-12

[25] Andryieuski A, Menzel C, Rockstuhl C, Malureanu R, Lederer F and Lavrinenko A 2010 Homogenization of resonant chiral metamaterials Phys. Rev. B 82235107

[26] Poddubny A, Iorsh I, Belov P and Kivshar Y 2013 Hyperbolic metamaterials Nat Phot. 7 948-57

[27] Shekhar P, Atkinson J and Jacob Z 2014 Hyperbolic metamaterials: fundamentals and applications Nano Converg. 14:1-17

[28] Kidwai O, Zhukovsky S V. and Sipe J E 2012 Effective-medium approach to planar multilayer hyperbolic metamaterials: Strengths and limitations Phys. Rev. A $\mathbf{8 5} 053842$

[29] Avrutsky I, Salakhutdinov I, Elser J and Podolskiy V 2007 Highly confined optical modes in nanoscale metal-dielectric multilayers Phys. Rev. B 75241402

[30] Kidwai O, Zhukovsky S V and Sipe J E 2011 Dipole radiation near hyperbolic metamaterials: applicability of effective-medium approximation. Opt. Lett. 36 2530-2

[31] Zhukovsky S V, Kidwai O and Sipe J E 2013 Physical nature of volume plasmon polaritons in hyperbolic metamaterials. Opt. Express 21 14982-7

[32] Zhukovsky S V., Andryieuski A, Sipe J E and Lavrinenko A V. 2014 From surface to volume plasmons in hyperbolic metamaterials: General existence conditions for bulk high-k waves in metaldielectric and graphene-dielectric multilayers Phys. Rev. B 90155429 
[33] El-Haija A J A 2003 Effective medium approximation for the effective optical constants of a bilayer and a multilayer structure based on the characteristic matrix technique J. Appl. Phys. 932590

[34] Rytov S M 1956 Electromagnetic properties of a finely stratified medium Sov. Phys. JETP 2 466-75

[35] Sheinfux H H, Kaminer I, Plotnik Y, Bartal G and Segev M 2014 Subwavelength Multilayer Dielectrics: Ultrasensitive Transmission and Breakdown of Effective-Medium Theory Phys. Rev. Lett. 113243901

[36] Andryieuski A, Malureanu R, Biagi G, Holmgaard T and Lavrinenko A 2012 Compact dipole nanoantenna coupler to plasmonic slot waveguide. Opt. Lett. 37 1124-6

[37] Markovich D L, Andryieuski A, Zalkovskij M, Malureanu R and Lavrinenko A V. 2013 Metamaterial polarization converter analysis: limits of performance Appl. Phys. B 112 143-52 


\section{Acknowledgements}

A.A. acknowledges financial support from the Danish Council for Independent Research via the GraTer project (Contract No. 0602-02135B). S.V.Z. acknowledges financial support from the People Programme (Marie Curie Actions) of the European Union's 7th Framework Programme FP7-PEOPLE-2011-IIF under REA grant agreement No. 302009 (Project HyPHONE). The authors also acknowledge H.H. Sheinfux for useful discussions. 